\title{
Quantitative nanomechanical IR spectroscopy for label-free molecular recognition
}

\author{
$\underline{\text { Seonghwan Kim }}$ \\ University of Calgary, 2500 University Dr. NW, Alberta T2N 1N4 Canada \\ sskim@ucalgary.ca
}

\begin{abstract}
:
Conventional infrared (IR) spectroscopy has been widely utilized in various applications since it can selectively detect target molecules using molecular vibrational characteristics. Although IR spectroscopy is an attractive technique for label-free recognition of target molecules, usually it is not very sensitive. Recently, we have developed quantitative nanomechanical IR spectroscopy using thermomechanically sensitive bi-material microcantilever in order to achieve high selectivity as well as sensitivity in label-free molecular recognition of target molecules [1]. Here, we present the various methods to enhance the performance of nanomechanical IR spectroscopy using a nanostructured microcantilever by controlling thermomechanical properties, surface to volume ratios, and even optical cavity structures [25]. Nanostructured microcantilevers with plasmonic absorbers are fabricated by coating gold layers onto nanoporous anodic aluminum oxide (AAO) cantilevers. Adsorbed explosive molecules such as trinitrotoluene (TNT), cyclotrimethylene trinitramine (RDX), and pentaerythritol tetranitrate (PETN) onto the cantilever generate nanomechanical IR spectroscopic responses. These nanomechanical IR spectra enable us to perform quantitative IR spectroscopy with surface adsorbed target molecules.
\end{abstract}

Key words: Nanomechanical IR Spectroscopy, Microcantilever, Label-free Sensor, Explosive, AAO

\section{References}

[1] S. Kim, D. Lee, X. Liu, X., C. Van Neste, S. Jeon, $\mathrm{T}$. Thundat, Molecular recognition using receptorfree nanomechanical infrared spectroscopy based on a quantum cascade laser, Scientific Report 3, 1111 (2013).

[2] D. Lee, S. Kim, S. Jeon, T. Thundat, Direct detection and speciation of trace explosives using a nanoporous multifunctional microcantilever, Analytical Chemistry 86, 5077-5082 (2014).

[3] D. Lee, O. Zandieh, S. Kim, S. Jeon, T. Thundat, Sensitive and selective detection of hydrocarbon/water vapor mixtures with a nanoporous silicon microcantilever, Sensors and Actuators B: Chemical, 206, 84-89 (2015).

[4] I. Chae, D. Lee, S. Kim, T. Thundat, Electronic Nose for Recognition of Volatile Vapor Mixtures Using a Nanopore-Enhanced Opto-Calorimetric Spectroscopy, Analytical Chemistry 87, 71257132 (2015).
[5] D. Lee, I. Chae, O. Kwon, K.-H. Lee, C. Kim, S. $\mathrm{Kim}, \mathrm{T}$. Thundat, Plasmonic absorbers with optical cavity for the enhancement of photothermal/opto-calorimetric infrared spectroscopy, Applied Physics Letters 110, 011901 (2017). 